\title{
A Real Time Priority based SCHeduler for LOW RATE WIRELESS SENSOR NETWORKS
}

\author{
Sambhaji Sarode, Jagdish Bakal \\ Department of Computer Science \& Engineering, \\ GHRCOE, RTM University, Nagpur, Maharashtra, India \\ \{sambhajisarode, bakaljw\}@gmail.com
}

\begin{abstract}
This paper presents a priority approach for censorious real-time traffic which flows particularly for low data rate wireless sensor and actor network (LR-WSAN). In the recent years, the demand for low rate wireless data transmission has been increased drastically in small scale industrial and non-industrial applications. The different traffic flows are increased by incorporating a variety of distinct sensing devices. In particular, injection of different traffic into network makes the communication system unstable and unreliable because of unnecessarily resource utilization, wasting energy for surplus delivery management and violation of time constraints. This paper aims to presents the new priority-based algorithm at actuator node for appropriate classification and categorization of data packets for delay sensitive and delaytolerant applications. A novel real-time priority-based scheduler (RTPS) is proposed to handle heterogeneous data flows simultaneously according to their transmission type. The priority-based data delivery is an essential research topic for low rate multi-event IEEE 802.15.4 sensor networks. A priority metric is designed to dynamically control various types of traffic based flows with considerations of packet type, delay, and buffer processing rate. TestBed results describe significant improvements in data reporting mechanism for delay sensitive and delay-tolerant applications over various topologies. The high priority transient traffic suffers less delay and presents effective packet delivery ratio compared with traditional approaches.
\end{abstract}

\section{KEYWORDS}

Transport protocol, Packet Scheduling, Reliability, Wireless sensor networks, WSN TestBed, Priority-based data reporting, IEEE 802.15.4 sensor networks, queue management, congestion control.

\section{INTRODUCTION}

This priority-based data reporting is an important task in industrial automation processes. Handling the multiple tasks [1]-[6] simultaneously increases the complexity of data transmission mechanism. A distributed coupon collection algorithm [4] is proposed to address the problem of delay using probabilistic model. It achieves the higher throughput. A channel access mechanism is designed based on the priority level in [5]. Nowadays [7]-[9] small scale industries have also started the automation process for improving the productivity and capability of managing the jobs remotely using small size short range devices. The periodic scheduling is useful in engineering applications to control and save an energy utilization of heterogeneous network [7] otherwise unnecessary waiting time hampers the network resources which shortens the system life. Thus, developing a priority-based data gathering mechanism is essential to handle large heterogeneous data simultaneously. This particular need has gained the attention of many active researchers in wireless sensor networks (WSNs) to develop an application specific packet scheduler over the MAC protocol. To evaluate the proposed protocol, a discrete event Markov chain model is developed. For example, Hypoxemia: a pulse oximeter, where reading ranges from 95 to 100 
International Journal of Computer Networks \& Communications (IJCNC) Vol.9, No.3, May 2017

percent. The value below 90 percent indicates low oxygen or need of external oxygen supply. It alerts the problem of breathing or circulation. In such applications, delivering the essential data [10], [11] timely to the doctor without a single packet loss is the crucial job of arterial blood gas sensor device. Nowadays sensor network is becoming the backbone of almost all types of application such as emergency, time constraint industrial controlling processes, and surveillance or observing events.

The current research not only focuses on the innovation in optimization algorithms but also motivates to develop the lightweight attribute aware schemes for specific events with hardware considerations. Sometimes researcher has to choose a little computing and longer life embedded devices purposefully for lightweight data delivery considering application requirements. RF application framework and routing protocols are designed by RF alliance whereas IEEE 802.15.4 community defines PHY \& MAC. The priority-based approach is presented over a normal realtime data transmission flows. Instead of treating distinct data flows equally at all the time, there is the need to design a flow based scheduling mechanism for delivering the sensor readings. Thus RTPS protocol is introduced to categorize the different traffic flows during in-network processing of a sensor network. Furthermore, it proposes the new priority metric for updating the priority type as it travels towards the sink; buffer processing rate is taken into consideration for measuring the congestion level of the buffer and additive-increase multiplicative-decrease (AIMD) method is used for renewing packet air rates. RTPS shows the less power consumption, optimal reporting rate, and high throughput, and less network latency as compared with First-Come-First-Serve method (FCFS) and Earliest Deadline First (EDF) method.

The strategies of the proposed work are briefed as follows.

- $\quad$ RTPS protocol presents the priority-based real-time data delivery approach using queuing operations efficiently. This protocol mainly delivers the higher traffic first over the regular traffic.

- A new priority weight metric is designed to allocate the priority dynamically at hop node in runtime which reduces the delay of long distance data packets.

- Auto-retransmission mechanism is modified and made it a flow based scheme to retry the lost packets in time to meet the deadline of time constraint applications.

- The mathematical model of RTPS protocol is implemented over nRF24L01 RF module (Nordic) with Arduino Nano-Atmega328 compatible) Testbed platform.

The flow of the paper goes as follows: The current research work overview and research gap identification are briefly discussed in part II. RTPS algorithm is introduced in part III for delivering higher priority information earlier than usual continuous information flows. Section IV, presents the real-time Testbed setup background, design consideration, and result in analysis and discussions. Finally, part V concludes and presents research directions.

\section{RELATED WORK}

A prime objective is to deliver essential information over underlying data delivery transmission protocols in the world of networking. However, considering the deadline and sensitivity of the information opens the dimensions for redesigning or inventing a new data transmission approaches for specific requirement of applications. The current reference work focus on the below aspects: 
International Journal of Computer Networks \& Communications (IJCNC) Vol.9, No.3, May 2017

- Preemptive and non-preemptive priority-based data reporting mechanisms

- Prioritized data packet scheduling over the MAC protocols.

- Priority-based scheduling using FCFS or EDF approach by managing single queues.

- Dynamic distributed priority updating mechanism over mesh hops during in-network processing. The transient and local traffic contributions are handled by considering their deadlines.

The transmission as mentioned earlier core operations of communication protocols plays an important role when traffic is significant in dense network wherein applications are less delay tolerant. In such situation utilizing the bandwidth effectively becomes the necessity. Therefore, after going through a literature review, it has been observed that work focus has been considerable gained an attention towards MAC layer core operations instead of managing ultimately at the transport or the application layer. The purpose is to decrease the waiting period of delay sensitive applications by avoiding unnecessary channel hearing. Moreover, the slot based TDMA medium access protocol plays a vital role in addressing this issue. Thus, the use of effective data scheduling algorithms with slot based TDMA approach prevents the signal interference and overheads for actual data transmission. In wireless sensor network, saving energy is an important challenge. Making communication reliable with less energy utilization is the need of industrial automation processes. In real time data flows, a delay is not significantly addressed yet. Industrial applications are having stringent timing requirements with different event data requirements simultaneously; therefore, developing a flow-aware data gathering mechanism is the necessity. This literature review mainly focuses on the existing MAC based data transmission mechanisms for LR-WSNs. This paper primarily focuses on real time and priority based data transfer. A cross-layer optimization or configuration approach (called ShedExGA) is introduced in [9]. A novel approach considers an application specific network constraints, namely, event reliability, data latency, reporting rate, service priority based differentiation. It does the micromanagement of cross-layer procedures like routing and channel specific controls. It manages to optimize the scheduling by minimizing the layering constraints.

However, two main approaches in Wireless HART networks are discussed in [10]. The local search algorithm is developed for optimal priority flow assignment for worst case analysis for given test schedulability. And the worst cases are investigated wherein the local search works optimally. It describes the optimal rate mechanism for managing the network dynamically. SMAC [12] uses periodic duty cycle for synchronizing sensor nodes together. It makes use of RTC/CTS to avoid the overburden of unwanted data transmission and helps to reduce the congestion. It saves the energy by preventing unnecessary data transmission overheads. D-MAC [13] works over the one way upstream based tree topology. It is an extended version of S-MAC to improve the congestion management in a high traffic situation. Hence, it reduces the latency due to the effective duty cycle. As compared with S-MAC, it increases the life of the network. TMAC [14] is equipped with effective flexible duty cycle mechanism. It is useful to reduce the listening period of the waiting traffic in the network. However, fixed duty cycle has worst side effects over an overall system like high latency and low throughput. Moreover, it uses immediate switching mechanism from listening state to sleeping state when no event occurs, or data is absent at a particular moment. Thus, results prove the less listening time against the fixed scheduling mechanism.

P-MAC [15] presents switching schedule for minimizing the energy consumption using preamble sampling approach. When no event reported then it immediately switches from hearing state into the sleeping state for the particular period, though it requires long preamble that wastes the network bandwidth. P-MAC follows the pattern schedule which is determined by following the duty cycle pattern of adjacent nodes. This protocol keeps itself into a sleep state for a longer period to preserve resources. Analysis illustrates less power utilization, however switching period 
International Journal of Computer Networks \& Communications (IJCNC) Vol.9, No.3, May 2017

needs to also taken into consideration. RAP protocol uses velocity operation to deliver high priority data over longer distance whereas Earliest Deadline First algorithm uses short deadline first approach to transport data to the desired destination. However, it does not give the guarantee of data classification and priority packet first. In PQMAC [16] classification of data is discussed. It does not ensure the data priority in non-uniform events. RF-MAC [17] presents the novel approach of charging nodes remotely through energy harvesting schemes. The energy transferring module is developed over the Mica2 hardware. In protocol design consideration, wherein nodes report the power level below a threshold; a high priority is given to energy delivery packets over the data delivery packets. It ultimately functions over the link layer. However, congestion management is not addressed. In [18] PriorityMAC protocol is introduced for priority based data transmission especially for critical traffic and periodic traffic in IWAN. It provides the service differentiation for higher priority traffic and reduces the latency and is implemented over the TelosB motes Testbed system. The traffic is categorized into four different classes, namely, TC1, TC2, TC3, and TC4 according to their priority levels, tolerance level, and access methods. At lower traffic levels, TDMA medium access approach is used whereas in TC2 class backoffindication approach is applied. It suffers from unfairness issue.

The CSMA/SF [19] presents the novel approach of priority-based traffic data packet first. It implements the length detection and anti-starvation mechanism by modifying the existing CSMA/CA protocol. The priority approach with multi-hop for linear wireless sensor networks (LWSNs) [20] demonstrates the collision-free data transmission. It works in two modes; namely, over straight line topology where only leaf node generates the traffic and delivers it over multiple hops; in the second mode, leaf and hop nodes generate the traffic over straight line topology. The nodes closer to sink nodes are given a high priority but for a shorter time. The different priority levels are assigned for contributing nodes depending upon hop distance away from sink node. An event-driven approach SIFT [21] MAC-based approach is presented. This strategy is applied to set of sensor nodes where the event occurs, and probability model is designed to deliver data with less collision, but unfortunately, early stage nodes follow bigger estimates for large node populations and hence small possibility for transmission. In real time TestBed emulation over 11 nodes and simulation support, transmission control protocol for media access present the adaptive rate control (ARC) mechanism for balancing power utilization and fairness of a sensor network. In [22], DBR MAC approach presents the high priority to key nodes. Sources whose contributions are greater called key nodes. Backoff time is used to reduce the congestion at key nodes, and more priority is given while accessing the channel. It uses cross-layer approach and delivers data via least hop distance route to floating base station precisely using a pressure gauge. In [23], a priority based data transmission protocol is designed for the multi-event sensor network. It is based on multiple buffers and scheduler is developed to handle the traffic flows. A FCFS mechanism is discussed for scheduling different traffic flows using packet scheduler in the multi-event sensor networks whereas in [24] EDF algorithm is applied for urban traffic management with considering the short deadline vehicle at various cross junctions of the road. In [25]-[27], a priority approach is designed for multi-event wireless sensor networks. It achieves the priority-based reliability based on the priority index of a packet and distance to reach the base station.

\section{RTPS PROTOCOL DESCRIPTION}

The various applications require chain-based topology setups like roadside infrastructure monitoring, oil gas pipeline, water tanks, and crop spaced over the straight line in the agriculture field. The objectives of RTPS are summarized as follows.

- To develop priority aware data reporting scheduler using data link layer protocol.

- To configure chain-based topology for protocol execution. 
International Journal of Computer Networks \& Communications (IJCNC) Vol.9, No.3, May 2017

The sensing device in the sensor field is called as reduced function device (RFD), and router/hop or a base station/ RF coordinator is defined as full function device (FFD). The RFD nodes are designed to sense, transmit, and receive and are less resource equipped nodes compared with FFD nodes. However, FFD nodes have more resource, and therefore they have additional processing overheads. The core network management operations consist of RTS/CTS mechanism for communication establishment before actual data transmission begins. It avoids hidden node problem during in-network processing. The reference topologies are described in figure 1. A few questions are considered to be answered through this research work as follows:

1) How would sensor select the neighboring node for data delivery?

2) What kind of channel access mechanism is appropriate for priority based data transmission?

3) How is hop updating the priority level of different traffic?

4) How will flexible retry mechanism help for preserving energy and reducing a delay of priority flow?

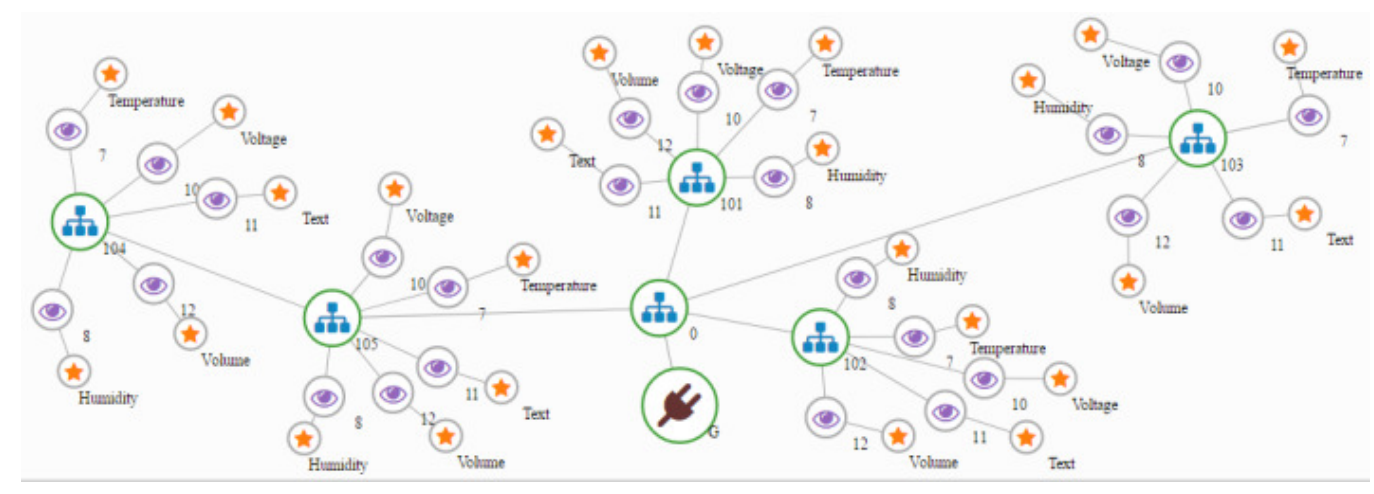

Figure 1. Multi-hop topological view captured during experimentation using sensor cloud solution to analyze the node and their sensor connectivity

A network consists of RF end devices (ED), RF router device (RD), RF Coordinator, and sensing area. Each ED performs information sensing and transmission to its upstream RD. A routing device performs data transmission based on the level of priority whereas sometimes it also acts as a relay node for delivering only received data. The RF Coordinator computes the reliability of each flow separately and generates positive or negative acknowledgment based on packet sequence number. The beaconless shock-burst data link protocol is used to implement the priority queue. The queuing operations are designed from priority event perspective. The first preference is given to delay sensitive activities at each RD device. The aim is to serve them in time by considering their sensitivity level. The proposed scheduler is tested over multi-hop topology with a finite number of nodes. While setting up the topology, nodes are placed randomly far from each other. Our proposed work differentiates concerning priority approach of data transmission compared with existing work. It provides 1) heterogeneous traffic based priority assignment, 2) priority renovation, and 3) flexible flow based auto retransmission.

The operations of priority queue are defined with considering its level dynamically. A threshold level $(l)$ is set to ensure the incoming packet flow is under control. Based on the queue status at various RD devices, the reporting rate is updated. This priority queuing approach helps to prevent the congestion in time and saves the energy of the network. While scanning the packets, the scheduler checks the priority weight of each incoming data packets. A packet whose weight is 
higher selected first for transmission sequentially. When any particular packet is selected; its priority weight is updated before it delivers to next RD node. The priority weight considers the hop count i.e. distance to reach to the RF coordinator and propagation time i.e. from $\mathrm{ED}$ device to current device. This approach helps to serve the more sensitive data packets first over the regular monitoring traffic. In real time environment, taking a decision in right time is a non-trivial task for critical events. Therefore, this metric has been designed to address the long distance priority traffic first which get hampered with newly sensed traffic at RD devices. Hence, the priority metric is designed considering the distance in terms of hop count away from the base station and propagation time (i.e. delay) taken from origin to current node. The priority metric is expressed in Equation 1.

$$
p_{w t}=\frac{p_{w t} h_{c}}{\omega d}
$$

The real values of hop count and delay bring the dynamism into the selection of each packet from the priority queue. Equation 1 computes the new priority weight value to each outgoing packet at router node. The purpose is to serve the long distance with sensitive data packet first over the regular traffic. The packet processing rate of queue ( $\left.p_{\text {rata }}\right)$ is defined as expressed in Equation (2). It indicates the saturation state or unsaturated state of the network. It helps to find the system performance concerning root causes of lost packets and successfully delivered packets.

$$
p_{\text {rate }}=\frac{\mu_{\text {out }}}{\lambda_{\text {in }}} \leq 1
$$

The difference between the current level and threshold limit is shown in equation 3. $\Delta T$ is an indicator of the queue. It is close to means; it shows the probability of the optimal state.

$$
\Delta T=q_{\text {size }}-\eta ; \frac{2.5}{q_{\text {size }}}>\Delta T>1 / 3 q_{\text {size }}
$$

The end to end time includes node processing time and traveling time in-between nodes. It gives the overall time during in-network processing data packets i.e. from source to the sink node.

$$
t_{\text {etoe }}=\sum_{h=1}^{m} t_{\text {proc }}^{h}+\sum_{l=1}^{n} t_{\text {pro }}^{n}
$$

The additive increase and multiplicative decrease mechanism (AIMD) have been used for making the data reporting traffic aware during in-network processing. If hop node detects that the queue level is being exceeded or packet loss is reported due to queue overflow event; then hop node immediately generates the control packet to sink. Sink takes a decision of change in reporting rate. An additive increase method is mentioned in Equation 5 which increases the air data rate when packet level in the buffer is detected low. When the performance is detected low due to low data rate, additive increase method (AI) increases the reporting rate to achieve higher system throughput.

$$
r_{i+1}=r_{i} \alpha
$$

The multiplicative decrease method (MD) is expressed in equation 6 which exponentially decreases the air data rate to control the buffer overflow. Whenever congestion is detected, the 
multiplicative method exponentially reduces the reporting rate to make the network congestion free.

$$
r_{i+1}=r_{i}^{\beta}
$$

A priority node for getting chance during scheduling process is

$$
P=N P(1-P)^{N-1}
$$

Where $\mathrm{P}$ indicates probability of packet delivery of high precedence packet and $\mathrm{N}$ indicates the number contributing nodes.

Table 1. Summary of mathematical notations

\begin{tabular}{|c|l|c|l|}
\hline Term & Definition & Term & Definition \\
\hline \hline $\mathrm{T}_{\text {type }}$ & Traffic flow type & $\omega$ & tuning parameter $(0.03)$ \\
\hline$\eta$ & packet level of a queue & $\mathrm{t}$ & queue threshold level \\
\hline $\mathrm{P}_{\mathrm{wt}}$ & Priority weight & $\lambda_{\text {in }}$ & incoming packet rate \\
\hline $\mathrm{h}_{\mathrm{c}}$ & hop count & $\mu_{\text {out }}$ & outgoing packet rate \\
\hline $\mathrm{d}$ & Delay (seconds) & $\mathrm{m}$ & Number of hops \\
\hline $\mathrm{n}$ & Number of links & $t_{\text {proc }}^{\text {n }}$ & Processing time at hop \\
\hline$a, \beta$ & Tuning parameters $(1.1,0.9)$ & $t_{\text {pro }}^{n}$ & Propagation time between nodes \\
\hline
\end{tabular}

The RTPS algorithm describes the traffic scheduling mechanism for simultaneously occurring events in the supervised sensing area. The single queue is used for storing the packets. A threshold value is set to queue to prevent the packet loss in an overflow condition. The $2 / 3$ packet level is the threshold value of the priority queue. The backpressure message is used to inform the packet level status in the queue. This approach helps well in advance to avoid unnecessary packet transmission and energy consumption. A data packet structure is restructured for different transfer flows in WSNs. For classification of data packets, additional filed are added into the basic data

\begin{tabular}{|c|c|c|c|c|c|c|c|}
\hline \multirow{2}{*}{$\begin{array}{l}\text { PP-1 } \\
\text { byte }\end{array}$} & \multirow{2}{*}{$\begin{array}{c}\text { Address } \\
\text { Field } 5 \\
\text { bytes }\end{array}$} & \multirow{2}{*}{$\begin{array}{c}\text { Packet } \\
\text { control } \\
\text { field }\end{array}$} & \multicolumn{4}{|c|}{ Payload (32 bytes) } & $\mathrm{CRC}$ \\
\hline & & & timestamp & $\begin{array}{c}\text { Priority } \\
\text { bit }\end{array}$ & $\begin{array}{l}\text { Hop } \\
\text { count }\end{array}$ & Data & byte \\
\hline
\end{tabular}
packet structure as shown in figure 2 .

Figure 2. Data Packet Structure of RTPS for priority-based data packet scheduling

A priority bit, hop count, and timestamp attributes play important role in data classification and scheduling phase of the RTPS data communication protocol. To check the packet error, a CRC filed is also taken into consideration during the experimentation. 
International Journal of Computer Networks \& Communications (IJCNC) Vol.9, No.3, May 2017

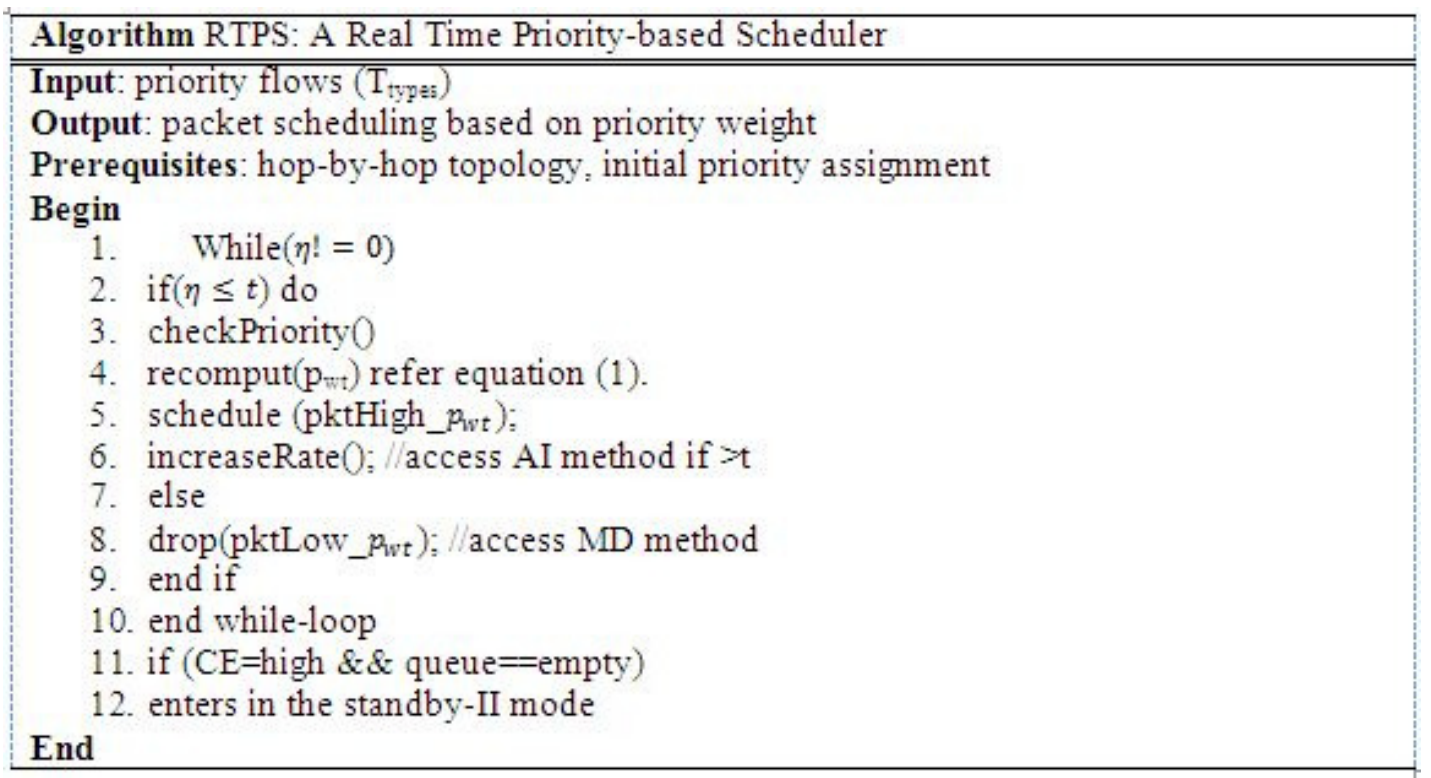

\section{RTPS SETUP \& EVALUATION}

We designed the TestBed using the NORDIC 2.4GHz nRF24L01 RF module [28] and ATmega 328 Arduino Nano [29] for evaluating the proposed work. It uses ISM band and operates over 2.4 - 2.4835GHz. It supports maximum 2Mbps air data rate. A standard configuration of IEEE 802.15.4 MAC is considered for experimental setup. However, we set 250kbps air data rate for the proposed protocol evaluation. The module has implicit core operations like automatic packet handling, auto-acknowledgement, selective auto retransmission and 6 logical data pipes are used and also, priority weight information is added into it.

The data pipe is enabled using EN_RXADDR register. Out of six data pipes, one data pipe becomes active and receives the packet. This feature is called as multiceiver. Figure 3 shows the actual end device used for experimentation. The same node hardware is used for hop node and RF coordinator. All have used shielded battery backup during emulation. The elapsed time is $250 \mu s$. It was chosen due to ultra lower power consumption feature. This module has $11.3 \mathrm{~mA}$ transmit power, with $0 \mathrm{dBm}, 12.3 \mathrm{~mA}$ receiver power, and packet pace is $2 \mathrm{Mbps}$, and $900 \mathrm{nA}$ in power down, $22 \mu \mathrm{A}$ at standby mode, and operates over the 1.9 to $3.6 \mathrm{~V}$ power supply range. It takes maximum $1.5 \mathrm{~ms}$ to wake from power down mode and 130us start-up time from standby mode. A delay bound is set to 0.2 seconds. It uses GFSK modulation technique. We have used $16 \mathrm{MHz}$ crystal frequency to manage the voltage level. The automatic packet handling includes flexible payload ( 25 bytes), dynamic data accumulation, dynamically data confirmation, and automatic packet splitting. Figure 3 shows the operational flow of the routing node. It is a heart of the RTPS protocol. Different functions like classification, sorting, priority revision, and scheduling are performed by the hop node. 


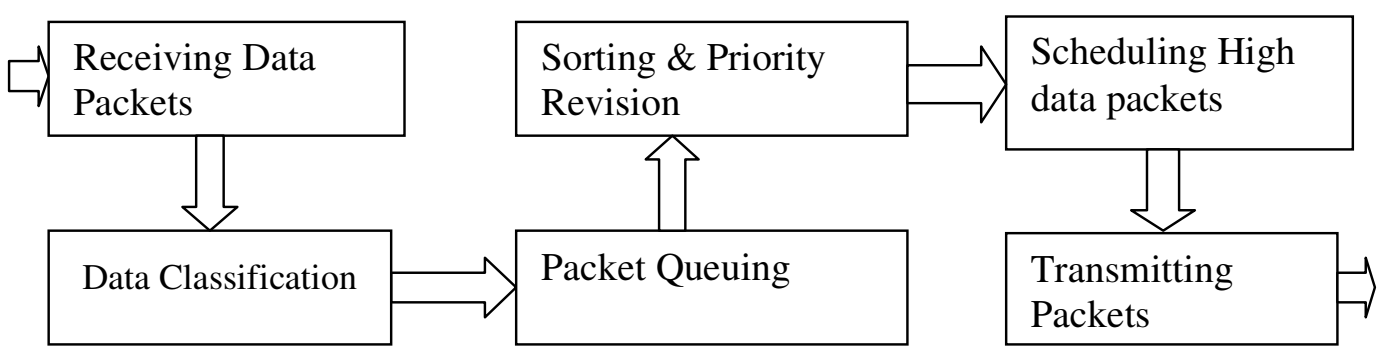

Figure 3. Block diagram of Operational flow Routing Node for scheduling high priority data packets

A RTPS uses FCFS mechanism and EDF approach [24]. It is modified according to the proposed priority approach. To authenticate results both scheduling approaches are put into service and compared with each other for delivering the real-time data packets. The packet structure is defined in figure 1. Due to variable payload feature, we have utilized 4 bytes payload for storing the temperature, humidity, accelerator, and heart beat counting. The packet size is 32 bytes in total. The network is setup with $8 \mathrm{RF}$ battery powered source nodes with one RF controller equipped with main supply. Each source node except leaf source node(s) senses its data and transmits the same if it is received from its downstream source node called as a repeater node. The real experimental view is depicted in figure 4 .

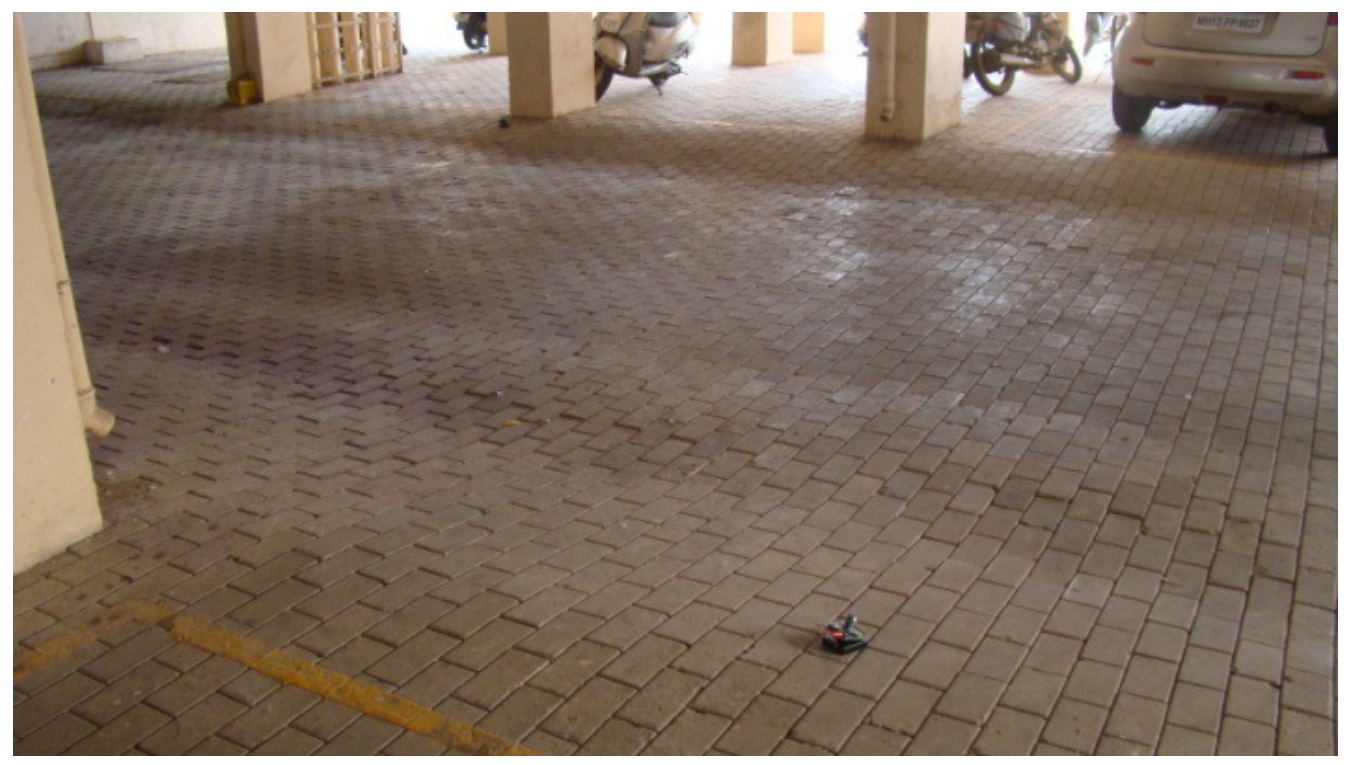

Figure 4. Experimental field view captured when nodes were equipped with 3 hop topology with heterogeneous sensing devices

They are generated by each RF end device separately. We have provided one sensor to each device distinctly to produce different traffic so that RF routing device will be able to get enough traffic at a time for classification dynamically. The priority bit information and hop counts are used at each RF routing device for revising the priority weight of each outgoing packet. 
International Journal of Computer Networks \& Communications (IJCNC) Vol.9, No.3, May 2017

\subsection{Real Time Analysis using Sensor Cloud Solution}

It is designed to monitor the status of each sensor device through this cloud solution in real time. Each active device could be controlled using various analytical tools which show the current reading through graphical representation, history of each sensor, battery level, the number of packets received, the path used, packet indication using beep, and also various filtering equation would be used to get the desired outcome. This solution saves the time of analysis and also gets to know the status of each device. It is easy to address the failure in time by monitoring the sensor activities. The end device could be equipped with many sensors because it assigns the unique identity to each sensor attached to a particular node. Therefore, we have generated multiple event data at each sensor node and which help us to create significant traffic to test priority queue efficiently. It is observed that queuing operations work efficiently for handling the different flows simultaneously. The data packets are monitored using the cloud solution, as shown in figure 5. The battery levels of an active node are monitored using group sensors as depicted in figure 6 .

\begin{tabular}{|c|c|c|c|c|c|c|c|c|c|}
\hline$\checkmark$ mysersens & MegD & Timetsme & Mose & Noste & Sensor & Type & ack & suetype & Messige \\
\hline \multirow{4}{*}{ 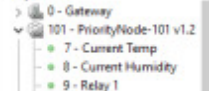 } & 6495 & 13/04/2017 4:13:29 & $\mathrm{RX}$ & 104 - Priserit, Node 104 & 12. Wonter Level & C.SET & No & v_voume & 0 \\
\hline & 6497 & 11/04/2017 4:12:28 & ax & $10 Q-$ Prieritytidode- 108 & 12- Woteritenel & CSET & no & v.voume & o \\
\hline & 699 & 11/04/2017 14.1829 & $\mathrm{RX}$ & 104 - PriserithNode 104 & 10. Vehage & C.SET & No & v_voLtAGE & 42 \\
\hline & 6501 & 13/6//2017 14:13:20 & $\mathrm{RX}$ & 102 - PrierititNode- 162 & 10-Vehtage & C.SET & No & v_vottace & 4.3 \\
\hline$\therefore 10$ - Vatuge & CNOS & 13/04/2017 141829 & $R X$ & 104 - Priberity Node- 104 & 11- Time & CSET & No & $V_{-} T E X T$ & 1680093107 \\
\hline - 11- Time & 6516 & $13 / 04 / 2017$ 14:12:20 & $\mathrm{RX}$ & 0 - Getenvey & NTERSWW & C.NTEENAL & No & LGATEWAY PEADV & 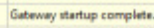 \\
\hline - 12- Water Level & ess & 11/04/2017 1413:29 & ax & 0 - Getenes & NTEFver & C.PRESENTATI & NNO & S_ABOUNNO REPEAT & 1.220-bets \\
\hline 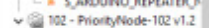 & 6521 & 13/04/2017 4:12:20 & $\mathrm{RX}$ & 101 - Prisity,Node- 101 & NTERSW & C.NTEENALL & No & LBATTERY LEVEL & 66 \\
\hline 7. 7. Cuentert Temp & 6523 & 1104/2017 41820 & $\mathrm{ax}$ & 101 - Prisentontode- 101 & 10-Vohpee & CSET & no & v_voctace & 40 \\
\hline - A- Current humidify & 6527 & 13/64/2017 $4: 12.20$ & $\mathrm{RX}$ & 101 - Prierity,Node 101 & 11- Time & CSET & No & V_tEXT & 206534550 \\
\hline$\because 9$ - Relay 1 & 6529 & 13/6/2017 14:020 & $a x$ & 103 - PrisititNode- 103 & 1- Cumere Humidity & CST & No & VHUM & 30 \\
\hline $\begin{array}{l}-10 . \text { Vothase } \\
\therefore \text { 11-Time }\end{array}$ & 6531 & $13 / 04 / 20174: 1329$ & $\mathrm{RX}$ & 103 - Priserit,Nede 103 & 12. Wonter Lenet & C.SET & No & v_vouue & 0 \\
\hline 12. Water Level & 653 & 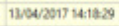 & $a x$ & 103 - Prisitititiode-16! & 10-Vohtage & CSET & No & v_votiace & 42 \\
\hline L S SADDUNO PEPEATER & 6535 & 11/04/2017 4413:29 & $\mathrm{ax}$ & 103 - Priserit, Nede 103 & NTERNAL & C.NTEENAL & No & LBATTERY LEVEL & $\pi$ \\
\hline 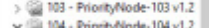 & 6539 & 13/4/2017 4:12:28 & ax & 103 - PrisititNode-163 & 11- Time & CSET & No & $V_{-}$_EXT & 10200031106 \\
\hline Y 104. Dionty Node 104 v1.2 & CSA & $13 / 04 / 20174,41029$ & $\mathrm{RX}$ & 101 - Pisionty Hede 101 & 11- Time & C.SET & No & V_tERT & Socosassso \\
\hline - 8-Current Humiday & 6543 & 13/46/2017 14:12:20 & RX & 103 - Prisity,tidode- 103 & 11- Time & C.SET & No & $V_{n}$ TEXT & 10202003100 \\
\hline$\because 9-$ Reltey 1 & eses & 13/04/2017 441829 & $\mathrm{RX}$ & 105 & 7-Cuniens Temp & CSET & No & $v_{-}$TEMP & NaN \\
\hline - 10 - votose & 6547 & 13/04/2017 14:13:20 & $\mathrm{RX}$ & 105 & 8. Cumeret Humisty & C.SET & No & V.HUM & -214729543 \\
\hline - 11- Time & esea & 19/6/2017 141320 & $\mathrm{ax}$ & 105 & 12- Woter Lend & CSET & no & v_voume & 0 \\
\hline 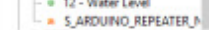 & 6551 & 13/04/2017 4:12:20 & $\mathrm{RX}$ & 105 & 10. Vethage & C.SET & No & v_VOLTACE & 4.5 \\
\hline 105 & toss & 11/04/2017 141620 & $a x$ & 105 & 11- Time & CSET & no & $V_{-T E T}$ & 130 \\
\hline
\end{tabular}

Figure 5. Screenshot of cloud interface for monitoring data and checking live sensing devices

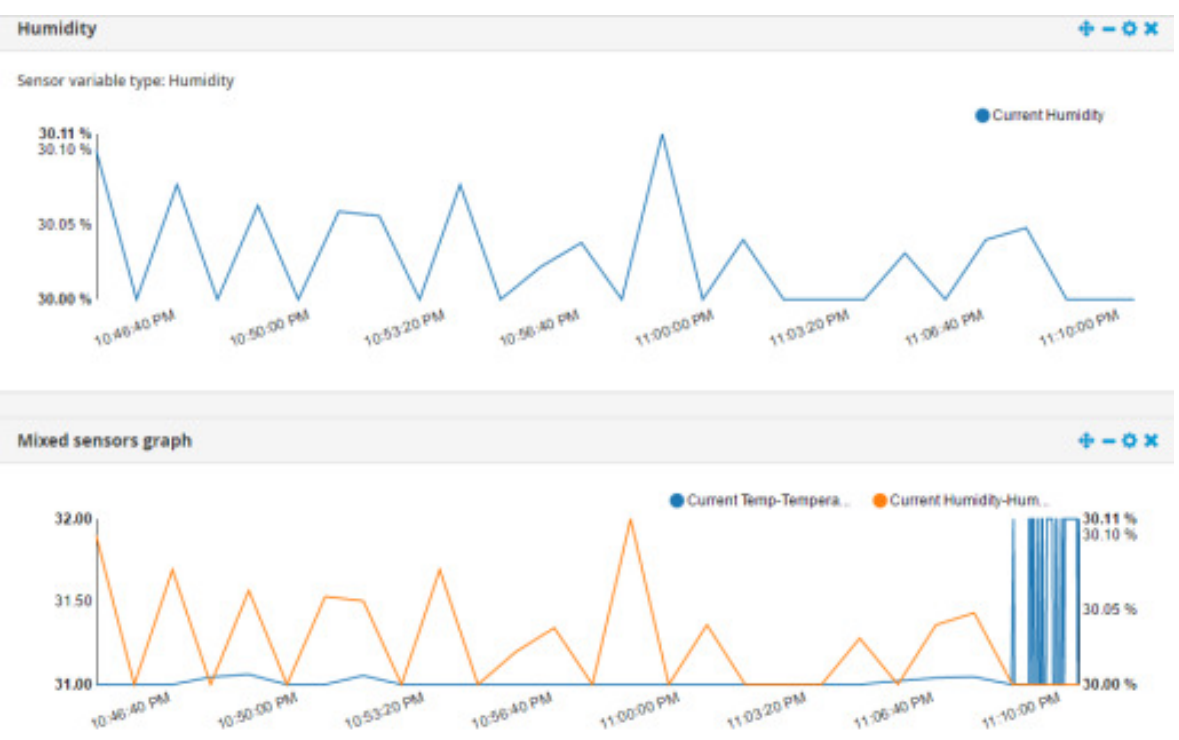

Figure 6. Screenshot of real-time energy consumption view of the source nodes 
The experiments are performed by varying the network topology, as described in secenario- 1 and 2. Scenario-1: in this section, we configured the system with maximum three hops and minimum two hops recorded. The performance of the proposed work is tested over different experimentation time. An average packet delivery ratio and throughput by varying the simulation time and packet interval time are rendered in figure 7 and 8 . An efficient queue management improves the packet delivery ratio, automatic retransmission for missed packets, and automatic path finding operation in case of link failures.

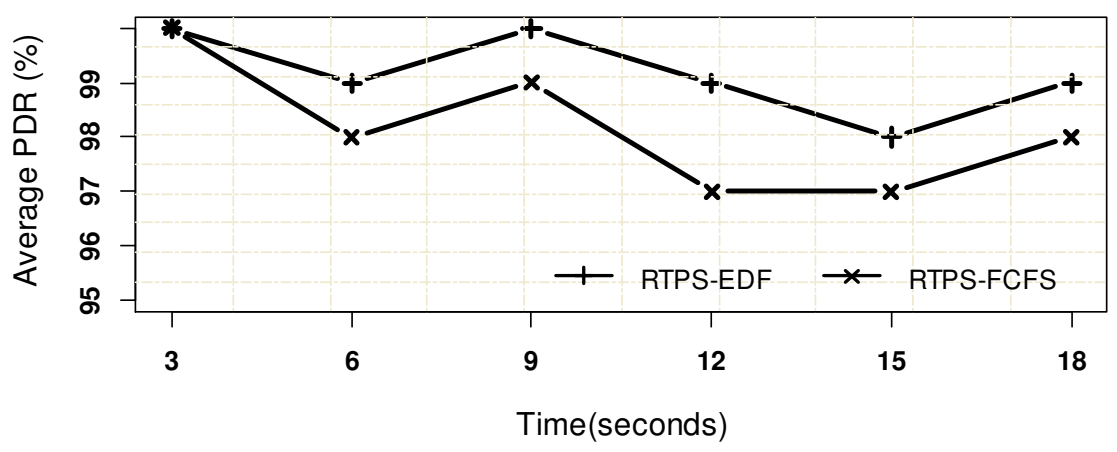

Figure 7. (scenario-1) shows the analysis of packet delivery ratio over a different period

The result indicates that many packets are received by the RF coordinator using RTPS approach compared with FCFS mechanism are more. The PDR is improved on an average 99\%, over average $96 \%$ against FCFS. It is achieved due to packet prioritization to sensitive sensor over regular sensors and effective queue management. Due to this approach, it is noted that the throughput is also significantly increased and the average throughput variation is observed around $6 \%$ higher than FCFS mechanism. The reporting rate is updated based on the state of a queue and has observed improvements with fewer packet drops. However, this protocol is tested over 8 nodes with 6 sensing devices. The observed PDR difference is small, but graph progress shows that when number source nodes increase with sensing devices, the difference is also proportionally increased.

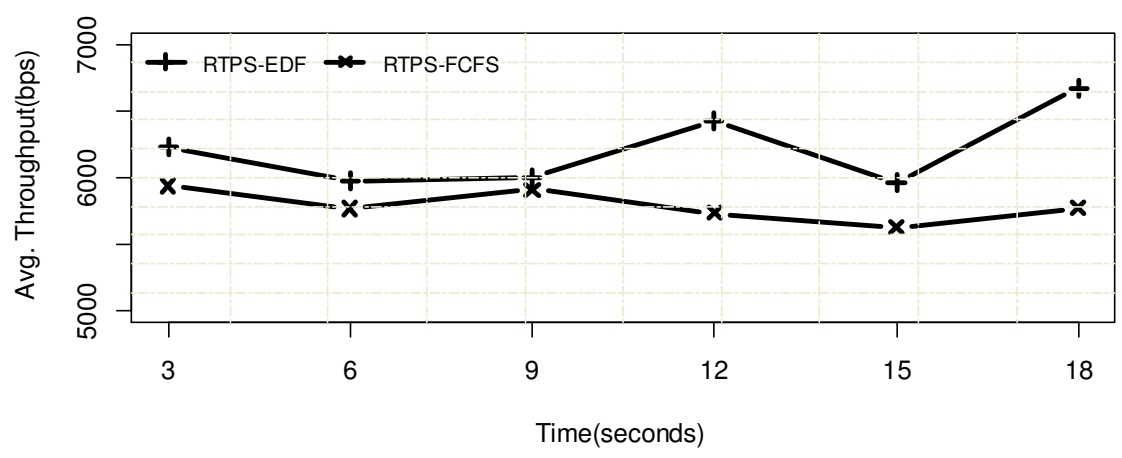

Figure 8. (scenario-1) shows the analysis of network throughput over a different time 
International Journal of Computer Networks \& Communications (IJCNC) Vol.9, No.3, May 2017

The average power usage and delay in diverse experimentation period is depicted in figure 9 and 10. The graph clearly describes that the RTPS consumes $12 \%$ less energy than the FCFS approach. This power consumption is because long distance packets are allowed first over the network. According to scenario-1, the delay is noted around 11\% less as compared with FCFS.

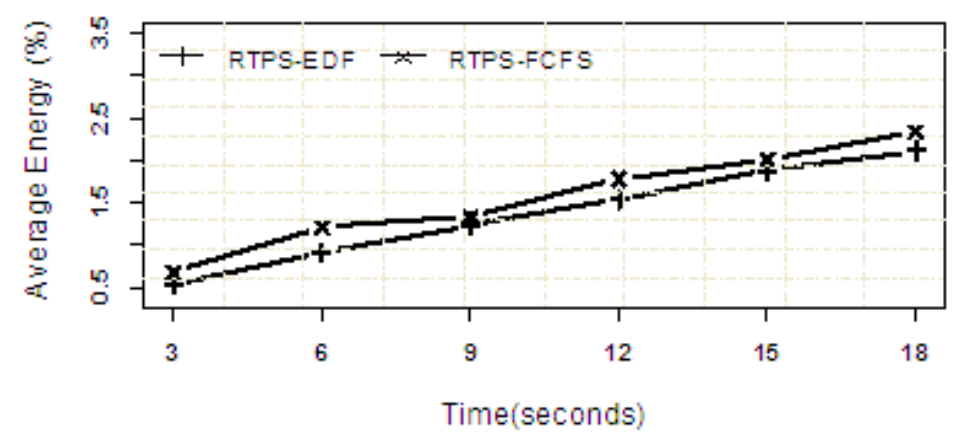

Figure 9. (scenario-1) shows the analysis of energy consumption over a different period

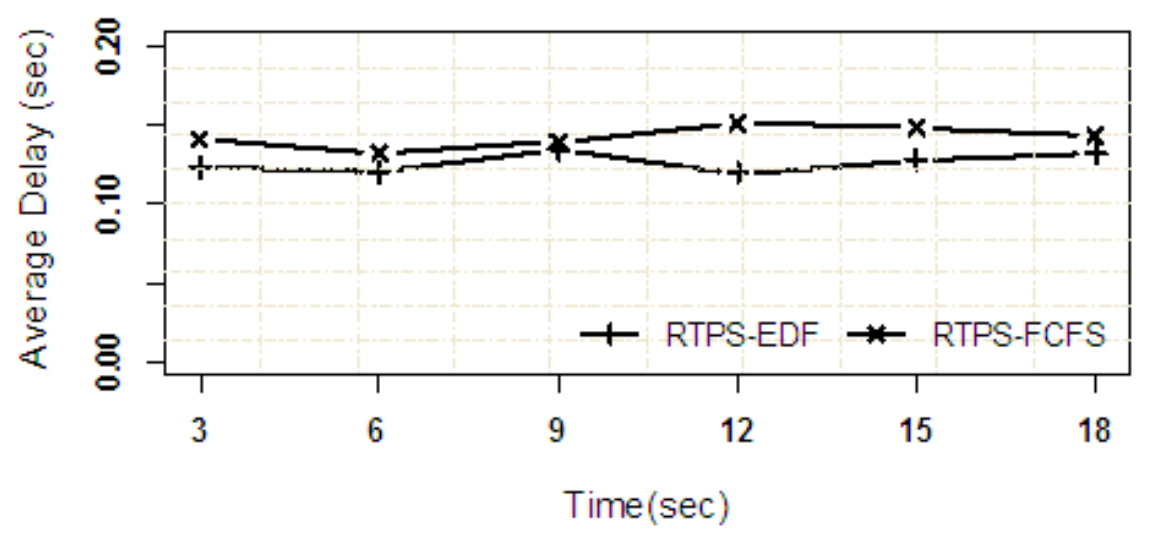

Figure 10. (scenario-1) shows the analysis of delay over a different period

Scenario-2: The performance of the presented work discussed over maximum recorded value of 5 hops and a minimum value of 2 hops. And with the variable inter-departure period, the same protocol setup is executed and observations regarding PDR, energy, delay, and throughput is discussed.

Thus it proves that the dynamism in priority weight metric helps to process the short deadline packets faster over newly sensed little weight packets. According to scenario-2, the average PDR ratio is noted approximately $99 \%$ over whereas the throughput is observed around $7 \%$ higher than FCFC method, shown in figure 11 and 12. 


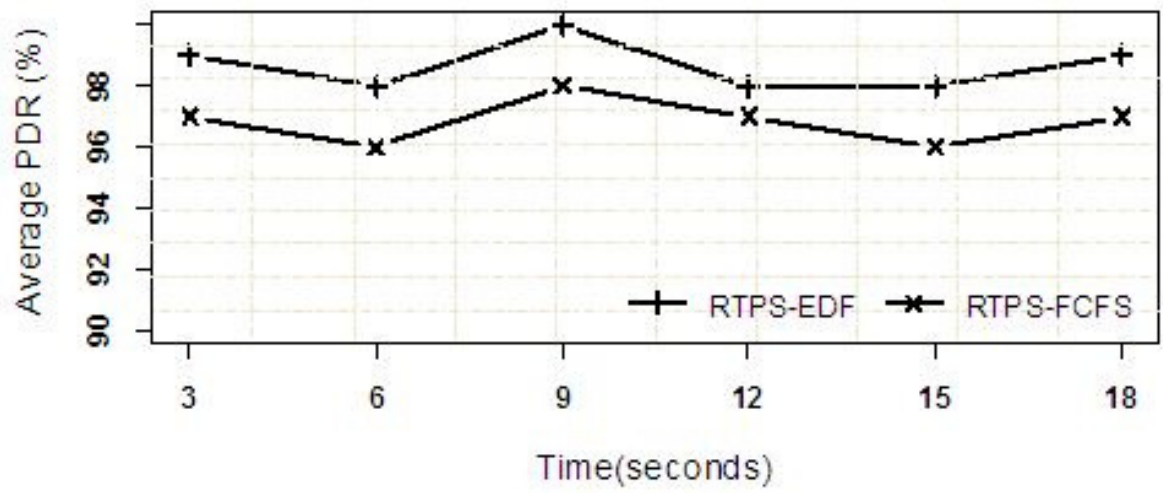

Figure 11. (scenario-2) shows the analysis of packet delivery ratio over a different period

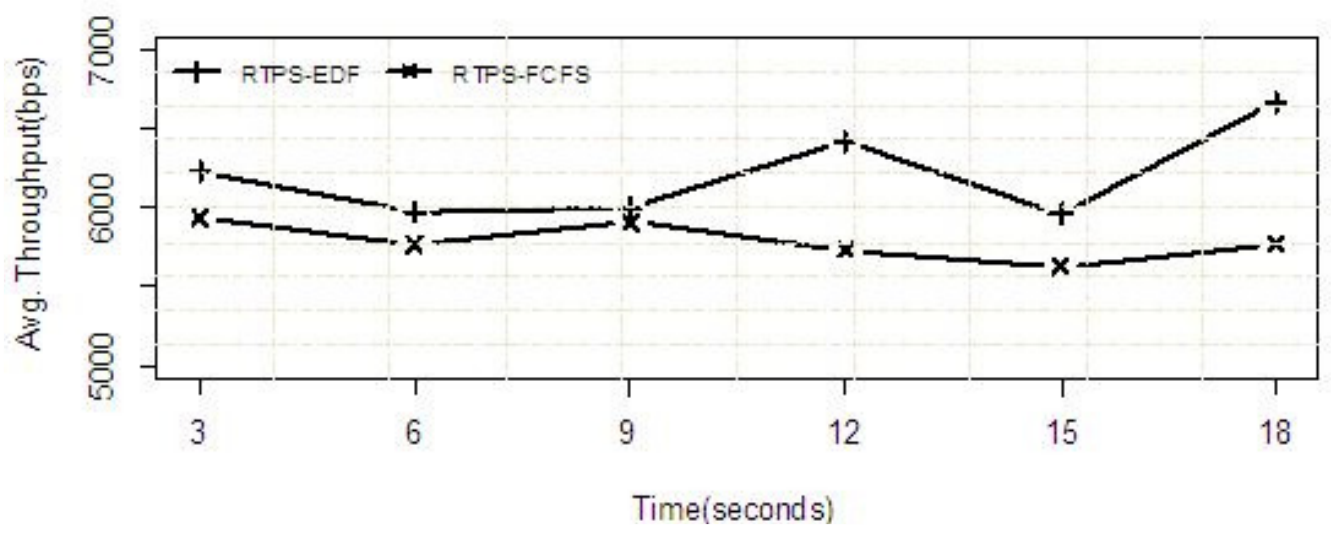

Figure 12. (scenario-2) shows the analysis of network throughput over a different time period

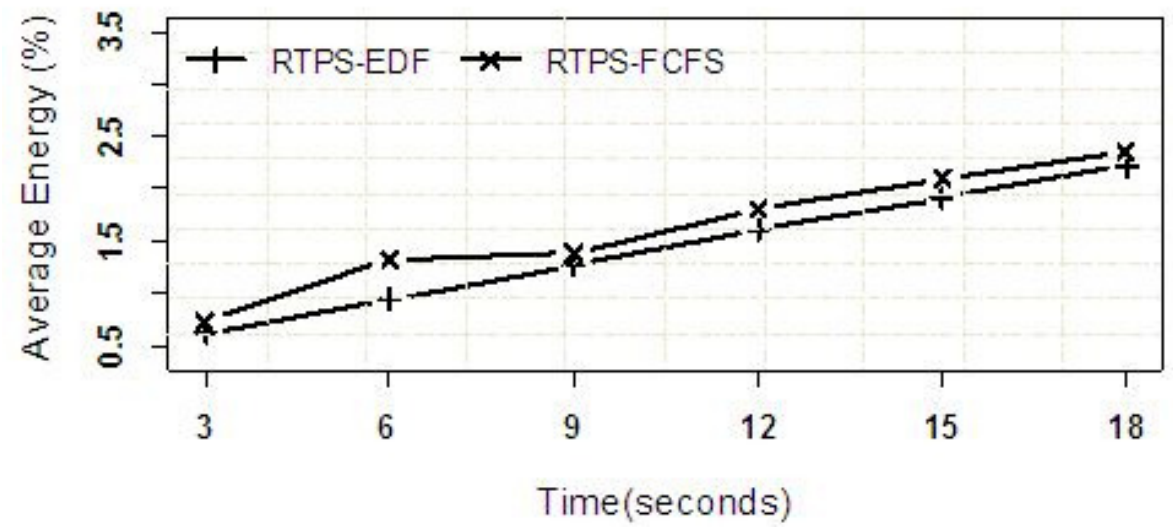

Figure 13. (scenario-2) shows the analysis of energy consumption over a different time period

Furthermore, this is achieved due to less retransmission of long distance packets. The RTPS schedules the short deadline packets first and prevents the packet loss because of deadline expire. Though the processing overheads are incorporated into repeater node; but it does not degrade the performance because processing time is minimal than retransmission time overheads. Thus 
reduces the propagation time on an average of each travelled packet in the network. The average $1.42 \%$ energy is spent and $11 \%$ less as compared with the FCFS mechanism and $6 \%$ delay is observed less compared with the FCFS method, as shown in figure 13 and 14.

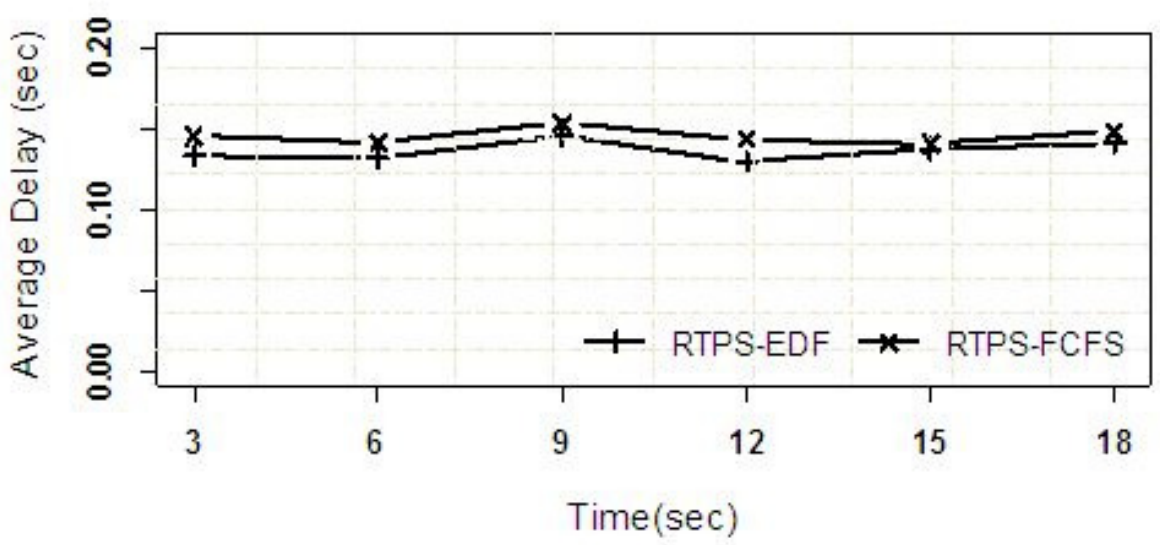

Figure 14. (scenario-2) shows the analysis of delay over a different time

It proves that the priority approach works better with EDF [20] algorithm for delivering the delay sensitive data packets. It reduces the chances of packet expiry due to deadline termination. The priority metric is designed with delay consideration, thus it not only increases the packet delivery ratio but also saves the overall life of the network. As a result, it shows the better system throughput against the traditional FCFS method. Therefore, RTPS can be used in time bounded applications such as heart beat counting, earthquake detection, fire alert systems, and much more.

\section{CONCLUding REMARKS}

In this work, we discuss the priority -based real-time transmission protocol, addressing the problems of flexible prioritization and variable rate differentiation. The priority is altered at various levels to reduce the packet loss of short deadline packets and to satisfy deadlines of sensitive packet first. In second part of the paper, RTPS performs the updating in the service rate differentiation. However, indirectly it contributes to reducing the delay of high priority traffic. Variable rate algorithm achieves target reliability and prevents the traffic jam in the successive interval of the network which results in less power utilization. Our proposed scheme shows $99 \%$ PDR and $12 \%$ less power usage as compared against FCFS method. In future, we plan to investigate the optimal operating frequency for higher priority classes and lower priority classes to function effectively. Besides, we plan to develop the beacon enabled priority-based MAC protocol for critical data delivery using interference free model.

\section{ACKNOWLEDGEMENTS}

The paper authors would like to take an opportunity to thank all reviewers for their valuable time for model discussion and constructive comments. We also would like to extend our thanks to my sensor open source forum community for providing help time to time. 
International Journal of Computer Networks \& Communications (IJCNC) Vol.9, No.3, May 2017

\section{REFERENCES}

[1] Chiara, Buratti \& Roberto, Verdone, (2016) "L-CSMA: A MAC Protocol for Multihop Linear Wireless (Sensor) Networks", IEEE Transactions on Vehicular Technology, Vol. 65, No. 1, pp 251-265.

[2] Fatima, Zahra, Djiroun \& Djamel, Djenouri, (2017) "MAC Protocols With Wake-Up Radio for Wireless Sensor Networks: A Review", IEEE COMMUNICATIONS SURVEYS \& TUTORIALS, VOL. 19, NO. 1, pp. 587-618.

[3] Elena,Uchiteleva, Abdallah, Shami, \& Ahmed, Refaey, (2017), "Virtualization of Wireless Sensor Networks Through MAC Layer Resource Scheduling”, IEEE SENSORS JOURNAL, VOL. 17, NO. 5, pp. 1562-1576.

[4] Chao, Li, Yongju, Xu, Chaonong, Xu, Zhulin, An, Boyu, Diao, \& Xiaowei, Li, (2016), "DTMAC: A Delay Tolerant MAC Protocol for Underwater Wireless Sensor Networks", IEEE SENSORS JOURNAL, VOL. 16, NO. 11, pp. 4137-4146.

[5] Tao, Zheng, Mikael, Gidlund, \& Johan, Åkerberg, (2016) "WirArb: A New MAC Protocol for Time Critical Industrial Wireless Sensor Network Applications", IEEE SENSORS JOURNAL, VOL. 16, NO. 7, pp. 2127-2139.

[6] Emanuele, Toscano \& Lucia, Lo Bello, (2012) "Multichannel Superframe Scheduling for IEEE 802.15.4 Industrial Wireless Sensor Networks", IEEE Transactions On Industrial Informatics, Vol. 8, No. 2, pp 337-350.

[7] Steven,X. Ding, Ping, Zhang, Shen, Yin \& Eve, L. Ding, (2013) “An integrated design framework of fault-tolerant wireless networked control systems for industrial automatic control applications," IEEE Trans. Ind. Inf., Vol. 9, No. 1, pp 462-471.

[8] Khaldoun, Al Agha, Marc-Henry, Bertin, Tuan, Dang, Alexandre, Guitton, Pascale, Minet, Thierry, Val, \& Jean-Baptiste, Viollet, (2009) "Which wireless technology for industrial wireless sensor networks? the development of OCARI technology," IEEE Trans. Ind. Electron., Vol. 56, No. 10, pp 4266-4278.

[9] Felix, Dobslaw, Tingting, Zhang, \& Mikael, Gidlund, (2016) "QoS-Aware Cross-Layer Configuration for Industrial Wireless Sensor Networks", IEEE Transactions On Industrial Informatics, Vol. 12, No. 5, pp. 1679-1691.

[10] Abusayeed, Saifullah, You, Xu, Chenyang, Lu, \& Yixin, Chen, (2011) "Priority assignment for real-time flows in wireless heart networks," In: Proc. 23rd Euromicro Conf. on Real-Time Syst, pp 35-44.

[11] Alec, Woo \& David, E. Cullar, (2001) "A transmission control scheme for media access in sensor networks", In: Proc. of 7th annual international conference on Mobile computing and networking, Rome, Italy, pp 221-235.

[12] Wei, Ye, J. Heidemann \& D. Estrin, (2002) “An energy-efficient MAC protocol for wireless sensor networks", In: Proc. of 21st Ann. Joint Conf. IEEE Computer and Communications Societies (INFOCOM'02), pp. 1567-1576.

[13] Stefano, Basagni, Alessio, Carosi, \& Chiara Petrioli, (2004) "Sensor-DMAC: dynamic topology control for wireless sensor networks", In: Proc. of IEEE 60th Vehicular Technology Conference, VTC2004-Fall, pp 2930-2935.

[14] Tijs, van Dam \& Koean, Langendeon, (2003) "An adaptive energy-efficient MAC protocol for wireless sensor networks", In: Proc. 1st Int'l Conf. Embedded Networked Sensor Systems (SenSys'03), pp 171-180.

[15] Tao, Zheng, Sridhar, Radhakrishnan \& Venkatesh, Sarangan, (2005) "PMAC: an adaptive energyefficient MAC protocol for wireless sensor networks", In: Proc. of Parallel and Distributed Processing Symposium, 19th IEEE International, pp 65-72.

[16] Hoon, Kim \& Sung, Gi Min, (2009) "Priority-based QoS MAC protocol for wireless sensor networks", IEEE International Symposium on Parallel \& Distributed Processing, IPDPS.

[17] Yousof, M. Naderi, Prusayon, Nintanavongsa \& Kaushik, R. Chowdhury, (2014) "RF-MAC: A Medium Access Control Protocol for Re-Chargeable Sensor Networks Powered by Wireless Energy Harvesting”, IEEE Transactions On Wireless Communications, Vol. 13, No. 7, pp 39263937. 
International Journal of Computer Networks \& Communications (IJCNC) Vol.9, No.3, May 2017

[18] Wei, Shen, Tingting, Zhang, Filip, Barac \& Mikael, Gidland, (2014) "PriorityMAC: A Priority enhanced MAC protocol for critical traffic in industrial wireless sensor and actuator networks," IEEE Trans. Ind. Informat., Vol. 10, No. 1, pp. 824-835.

[19] Guanhua, Wang, Kaishun ,Wu \& Linoel, Ni, (2014) "CSMA/SF: Carrier Sense Multiple Access with Shortest First", IEEE Transactions on Wireless Communications", Vol. 13, No. 3, pp. 16921702.

[20] Wei, Shen, Tingting, Zhang \& Mikael, Gidland, (2013) "Joint routing and MAC for critical traffic in industrial wireless sensor and actuator networks," In: Proc. of 2013 IEEE International Symposium on Industrial Electronics, pp. 1-6.

[21] Kyle, Jamieson, Hari, Balakrishnan \& Y.C.Tay, (2003) "Sift: A MAC protocol for event -driven wireless sensor networks”, Tech.Rep. MIT-LCS-TR-894, Massachusetts Institute of Technology, EWSN 2006, LNCS 3868, pp. 260-275.

[22] Hai, Zhijie, Jerry, Shi \& Jung-Hong, Cui, (2008) "DBR: Depth-based routing for underwater sensor networks," In: Proc. of NETWORKING Ad Hoc and Sensor Networks, Wireless Networks, Next Generation Internet. Berlin, Germany: Springer, pp 72-86.

[23] Sambhaji, Sarode, Jagdish, Bakal \& Latish, Malik, (2016) "Reliable and Prioritized Data Transmission Protocol for Wireless Sensor Networks", In: Proc. of the International Congress on Information and Communication Technology, Udaipur, India, Vol. 439, pp 535-544.

[24] Asif, Ahmad, Rabia, Arshad, Sahibzbada,. Ali Mahamud, Gul, M. khan, \& Hamed S, AlRaweshidy, (2014) "Earliest-Deadline-Based Scheduling to Reduce Urban Traffic Congestion", IEEE Transactions On Intelligent Transportation Systems, Vol. 15, No. 4, pp 1510-1526.

[25] Sambhaji, Sarode, Jagdish, Bakal, (2017) "Performance Analysis of Beacon enabled Prioritized CSMA/CA for IEEE Sensor Networks", International Journal of Applied Engineering Research, ISSN 0973-4562 Volume 12, Number 8 (2017) pp. 1622-1627.

[26] Sambhaji, Sarode, Jagdish, Bakal, (2017) "PriTLP: A Priority-based Transport Layer Protocol for Low Rate Wireless Sensor Networks", American Journal of Sensor Technology, American Journal of Sensor Technology, vol. 4, no. 1 (2017), pp 21-29.

[27] Sambhaji, Sarode, Jagdish, Bakal, (2017) "A Slotted CSMA/CA of IEEE 802.15.4 Wireless Sensor Networks: A Priority Approach”, International Journal of Computer Trends and Technology (IJCTT) - Volume 44 Issue 1, pp 33-38.

[28] http://www.nordicsemi.com/eng/Products/2.4GHz-RF.

[29] https://www.arduino.cc/en/Guide/ArduinoNano.

\section{Authors}

Sambhaji Sarode received BE and ME in information Technology from the SPPU University Pune in 2005 and 2010, respectively. Currently, he is working as an assistant professor in the Department of Computer Engineering, MIT College of Engineering Pune since Jan 2006. He is having $11+$ years of experience. He is currently working towards a Ph.D. degree in Computer Science and Engineering; RTM Nagpur University under the supervision of Prof. Dr. J. W. Bakal. His research interest includes reliability approaches in wireless sensor networks, Cognitive security, and the Internet of Things. He has completed two research funded projects. He has delivered many expert sessions in STTP programs and workshops to name a few "Use of Modeling in Network" in TEQIP-II STTP development program, "IoT: Sensor Network" in TEQIP-II STTP development program, etc. He is the author of three books and published many research articles in journals and conferences.

Jagdish Bakal received M. Tech. (EDT), from Dr. Babasaheb Ambedkar Marathwada University, Aurangabad. Later, He completed his Ph.D. in the field of Computer Engineering from Bharati Vidyapeeth University, Pune. He is presently working as Principal at the S.S. Jondhale College of Engineering, Dombivali (East) Thane, India. In the University of Mumbai, he was on honorary assignment as a chairman, board of studies in Information Technology and Computer Engineering. He is also associated as chairman or member of Govt. committees, University faculty interview committees, for interviews, LIC or various approval works of institutes. He has more than 27 years of academics experience
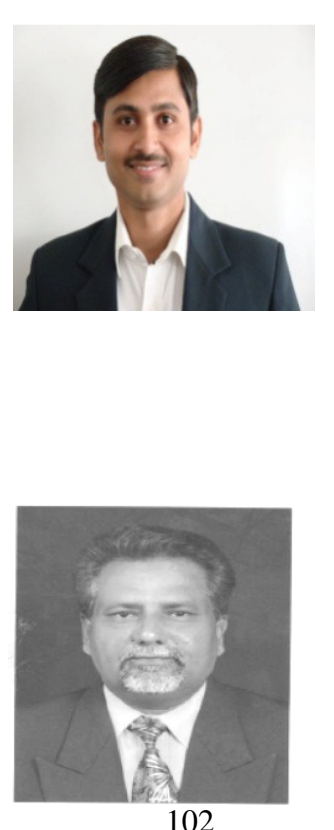
including HOD, Director in previous Engineering Colleges in India. His research interests are Telecomm Networking, Mobile Computing, Information Security, Sensor Networks and Soft Computing. He has publications in journals, conference proceedings in his credit. During his academic tenure, he has attended, organized and conducted training programs in Computer, Electronics \& Telecomm branches. He is a Professional member of IEEE. He is also a life member of professional societies such as IETE, ISTE INDIA, CSI INDIA. He has prominently contributed in the governing council of IETE, New Delhi INDIA. 